\title{
Comment on "Which Patients with Obesity Are at Risk for Renal Disease?"
}

\author{
Gioacchino Li Cavoli Calogera Tortorici Luisa Bono Angelo Ferrantelli \\ Vitalba Azzolina Tancredi Vincenzo Li Cavoli Angelo Tralongo
}

Nephrology Dialysis Renal Transplant Department, ARNAS Civico Di Cristina, Palermo, Italy

Dear Editor,

We read with interest the review of Dr. Sandino et al. [1]. We agree with the authors that the association between the obesity, nonnephrotic proteinuria, and endstage renal disease shows an increasing trend, but it remains unclear why only some subjects with obesity develop CKD. The wide spectrum of pathological renal changes in patients with metabolic syndrome (MS) justifies the heterogeneous course toward end-stage renal disease (Fig. 1,2). Probably there exists a relevant research gap in our knowledge base in the pathophysiology of obesity and the link with the chronic kidney disease [2]. The link between kidney disease and MS is investigated by experimental and human histopathology. Choung et al. [3], among 3,263 native kidney biopsies, have described a broad spectrum of renal histological lesions in patients with obesity, not only in the glomerulus but also in tubulointerstitial and vascular compartments. In this experience, there was a wide heterogeneity in the renal disease (diabetic nephropathy, acute tubular necrosis, hypertensive nephrosclerosis, IgA nephropathy, membranous nephropathy, and lupus nephritis) affecting this population. Longitudinal clinical studies in humans highlighted that glomerulomegaly is the histological characteristic change in glomerular hyperfiltration, and the increase in albuminuria is the first clinical manifestation. In human pathology, obesity is associated with CKD, and glomerulomegaly, proteinuria, and FSG define the obesity-related glomerulopathy (ORG). Histological studies have dem-

karger@karger.com

(C) 2021 S. Karger AG, Basel

www.karger.com/nef

Karger! onstrated that ORG encompasses a continuum from glomerulomegaly to glomerulosclerosis. On kidney biopsy, the patients with massive obesity develop glomerulomegaly. In the experience of Serra, among 95 obese patients with a mean BMI of $52 \mathrm{~kg} / \mathrm{m}^{2}$, the kidney biopsies reported a greater prevalence of glomerulomegaly, podocyte hypertrophy, mesangial cell proliferation, and an increase in mesangial matrix [4]. Okabayashi et al. [5] demonstrated in patients with a BMI $25-30 \mathrm{~kg} / \mathrm{m}^{2}$ typical histologic features of ORG (low glomerular density with glomerulomegaly). In 49 obese renal donors, compared to 41 nonobese controls, Rea reported a larger glomerular planar surface area; the urinary microalbumin excretion and the patient weight correlated with this finding, but the obese renal donors did not show evidence of glomerulosclerosis [6]. Alexander examined 146 patients undergone to elective nephrectomy for renal cell carcinoma. Twelve of these also had MS. Tubular atrophy, interstitial fibrosis, arterial sclerosis, global glomerulosclerosis, and segmental glomerulosclerosis had a higher prevalence in these patients with MS than healthy subjects, suggesting evidence of vascular damage, but the glomerular volume was not different between MS-patients and controls [7]. Kambham et al. [8] compared 71 patients with ORG and 50 patients with idiopathic FSG without ORG. The authors detected FSG with glomerulomegaly in 57 and glomerulomegaly alone in 14 of ORG patients and highlighted the significant overlap in clinical and pathologic features between ORG and idiopathic FSG. The relationship 


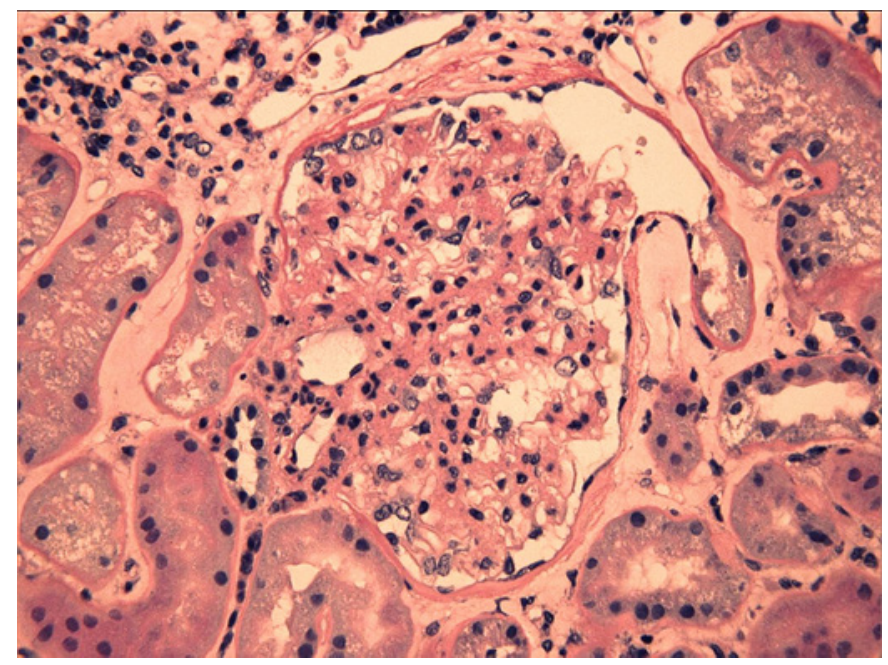

Fig. 1. Renal biopsy. Light microscopy: glomerulomegaly with arteriolar hyalinosis and perihilar segmental sclerosis with synechiae formation and diffuse thickening of capillary basement membranes $(\mathrm{HE}, \times 40)$.

between MS and nephropathy is complex. Despite the strong epidemiologic association, the renal involvement in MS has not been proven. A greater knowledge of the combination of FSG and glomerulomegaly in MS can help in the understanding of pathophysiological mechanisms of this new emergency. It would be interesting to design studies regarding the knowledge gap between the renal histology and MS, overweight and obesity.

\section{Acknowledgment}

We thank our colleagues at the Department of Nephrology for their help in the formation of this article.

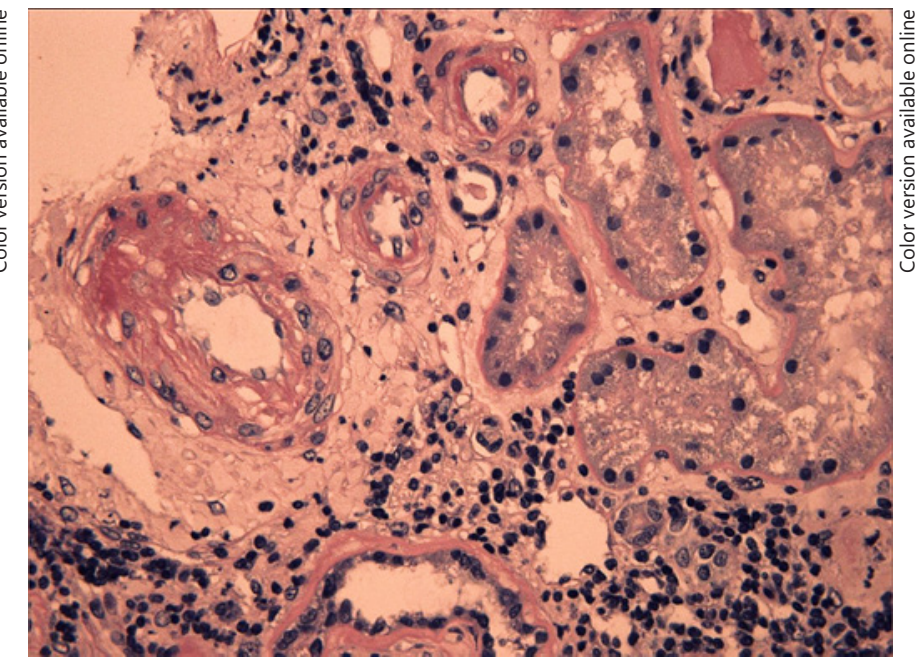

Fig. 2. Renal biopsy. Light microscopy: hyalinosis and marked thickening of medium and small arteries wall, due to intimal fibrosis with narrowing of the lumen, and inflammatory interstitial infiltrates with evidence of tubulitis $(\mathrm{HE}, \times 40)$.

\section{Conflict of Interest Statement}

The authors have no conflicts of interest to declare.

\section{Funding Sources}

The authors have no funding sources to declare.

\section{Author Contributions}

All the authors contributed equally to this study, revised the paper, and approved the final version of the manuscript.

\section{References}

1 Sandino J, Luzardo L, Morales E, Praga M. Which patients with obesity are at risk for renal disease? Nephron. 2021. doi: 10.1159/ 000513868.

2 Friedman AN, Kaplan LM, le Roux CW, Schauer PR. Management of obesity in adults with CKD. J Am Soc Nephrol. 2021 Feb 18; 32(4):777-90.

3 Choung HG, Bomback AS, Stokes MB, Santoriello D, Campenot ES, Batal I, et al. The spectrum of kidney biopsy findings in patients with morbid obesity. Kidney Int. 2019 Mar; 95(3):647-54
4 Serra A, Romero R, Lopez D, Navarro M, Esteve A, Perez N, et al. Renal injury in the extremely obese patients with normal renal function. Kidney Int. 2008;73:947-55.

5 Okabayashi Y, Tsuboi N, Sasaki T, Haruhara K, Kanzaki G, Koike K, et al. Glomerulopathy associated with moderate obesity. Kidney Int Rep. 2016 Aug 12;1(4):250-5.

6 Rea DJ, Heimbach JK, Grande JP, Textor SC, Taler SJ, Prieto M, et al. Glomerular volume and renal histology in obese and non-obese living kidney donors. Kidney Int. 2006;70: 1636-41.
7 Alexander MP, Patel TV, Farag YM, Florez A, Rennke HG, Singh AK. Kidney pathological changes in metabolic syndrome: a crosssectional study. Am J Kidney Dis. 2009;53:751-9.

8 Kambham N, Markowitz GS, Valeri AM, Lin J, D'Agati VD. Obesity-related glomerulopathy: an emerging epidemic. Kidney Int. 2001; 59:1498-509. 